\title{
ABANDONO DE TIERRAS Y CAMBIO CLIMÁTICO FRENTE A LA INNOVACIÓN EN EL SECTOR VITICOLA
}

\author{
$\underline{\text { Consuelo Calafat-Marzal }}{ }^{*}$, Mercedes Sánchez Garcíab ; Aurea Gallego-Salguero ${ }^{\mathrm{c}}$ \\ a Departamento de Economía y Ciencias Sociales. Universitat Politècnica de València \\ ${ }^{b}$ Departamento de Empresas. Universidad Pública de Navarra \\ ${ }^{c}$ Departamento de Departamento de Ingeniería Cartográfica, Geodesia y Fotogrametría. Universitat \\ Politècnica de Valencia.
}

\begin{abstract}
Resumen
El abandono de tierras agrícolas con actividad productiva y la despoblación de zonas rurales son fenómenos generalizados en toda Europa. El objetivo de este estudio es analizar los factores que más influyen en el mantenimiento o abandono del viñedo mediante un enfoque metodológico multinivel, comparando los efectos entre municipios en zonas de despoblación con las zonas que no presentan este fenómeno. Las variables utilizadas para el modelo distribuyen en tres niveles: parcelas, que identifican las características estructurales de las explotaciones; municipio, que describen las condiciones socioeconómicas y de cambio climático dónde se ubican las explotaciones; y mercado, que indican el comportamiento de los precios del sector. El estudio se lleva a cabo en la Comunidad Valenciana (CV), en una región española situada en el litoral mediterráneo, con elevados conflictos por el uso del suelo en el litoral, pero con elevado número de municipios rurales con riesgo de despoblación en el interior, en los que el viñedo es una opción de generación de rentas y fijación de población. Los resultados indican que la adaptación de innovación y el cambio técnico están relacionados con el mantenimiento de las explotaciones, y como el cambio climático incide en el abandono de cultivos en las zonas con mayor número de siniestros.
\end{abstract}

Palabras clave: Multinivel, cambio climático, despoblación, innovación sectorial

\section{Introducción}

El sector vitícola está extendido a lo largo de toda España, siendo un agente de desarrollo socioeconómico en muchas zonas. En las últimas décadas, el sector vitivinícola se ha visto sometido a una intensa globalización, competencia internacional e impacto del cambio climático, un hecho que plantea tanto retos como oportunidades a las regiones vitivinícolas, que se ven obligadas a adoptar estrategias innovadoras.

El régimen de derechos de plantación de viñedo sustituyó por el sistema de autorizaciones en enero de 2016, con vigencia hasta el 31 de diciembre de 2030, motivo por el cual la composición del potencial vitícola ha experimentado un cambio. En la Comunidad Valenciana (CV) las modificaciones no han frenado el descenso continuado de la superficie vitícola. De 1983 a 2019 la reducción de superficie de cultivo fue de 105.485 hectáreas de viñedo, siendo un descenso porcentual de $61,9 \%$, destacando los trece primeros años (1983-1995) donde se perdieron 71.870 hectáreas, debido en parte a las políticas de arranque del cultivo, y dejando la superficie de viñedo concentrada principalmente en zonas de Valencia y Alicante.

Las opciones que pueden ser elegidas por los viticultores para sus parcelas son: (1) abandono; (2) plantación (siempre que tuvieran derechos de plantación o los hubieran adquirido antes de la fecha límite para adquirirlos y la posterior liberalización); (3) arranque; (4) reestructuración y (5) mantenimiento de la explotación. Las opciones de plantación y arranque pretenden lograr un equilibrio entre la oferta y la demanda de vino de calidad, en cambio mediante la reestructuración se pretende aumentar la competitividad de los productores (CE, 2008). Si las condiciones locales de las parcelas de los agricultores no son propicias para una producción viable, también pueden optar por abandonarlas. El agricultor también puede optar por seguir como hasta ahora (una quinta alternativa).

En este contexto, las decisiones de los viticultores están condicionadas por la estructura de sus explotaciones (Montagut y Gogliotti, 2008), destacando las prácticas encaminadas a la innovación que permita una gestión más dinámica (Giannoccaro y Berbel, 2011), pero también a las de su entorno, de manera que las características socioeconómicas de los municipios en las que están ubicadas (Engler y Toledo, 2010; Naiggolan et al., 2013), y las acciones de la Política Agrícola Común (PAC), principalmente de los pagos que reciben los agricultores (Cortignani y Severini, 2011; Giannoccaro y Berbel, 2011). La relación de estos condicionantes con la decisión de los viticultores puede ser directa o inversa y, además, el grado de significación será distinto.

Para determinar el impacto en las decisiones de los viticultores se propone una metodología basada en los modelos multinivel multinomiales, que permiten establecer las relaciones entre las decisiones sobre las 
parcelas con sus características estructurales y las características socioeconómicas municipales y las ayudas de PAC, entendiendo que las parcelas vitícolas, y el contexto dónde se desenvuelven, municipios, son una estructura jerárquica donde las parcelas están aisladas en un plano individual y posteriormente establecidas en grupos de niveles superiores.

La hipótesis de partida del presente estudio es que existe una combinación de variables que influye en las decisiones de los viticultores, relacionada con la estructura de las parcelas (principalmente del tamaño de la explotación y las prácticas innovadoras), las características de su entorno, como son la pertenencia a municipios más o menos pequeños, con renovación de la población activa, principalmente del sector agrario, y, además, la contribución de las ayudas de la PAC al mantenimiento de las explotaciones.

\section{Objetivo}

El objetivo de este estudio es analizar la decisión de los viticultores (mantener la explotación, nuevas plantaciones, replantar, arrancar o abandonar) según las características estructurales de las explotaciones, los factores socioeconómicos municipales y las ayudas recibidas de la PAC mediante métodos multinivel.

\section{3. Área de estudio}

El modelo se ha aplicado al área de estudio de la CV (Figura 2), centrado en las comarcas principalmente vitivinícolas: La Plana de Utiel-Requena (Valencia), el Alto Vinalopó (Alicante y Vinalopó Medio (Alicante). En la comarca de la Plana de Utiel-Requena, compuesta por 9 municipios, se concentra el $70 \%$ de la superficie de viñedo de la provincia de Valencia (52.412 recintos con una superficie de 40.545,88 ha), donde el 99,7\% son de viñedo de uva para vino. En las comarcas del Alto Vinalopó y Vinalopó Medio, compuestas por 7 y 10 municipios respectivamente, concentran el 73,5\% de la superficie de viñedo de la provincia de Alicante (17.610 recintos con una superficie de $16.775,11 \mathrm{ha}$ ), donde $42,2 \%$ son de viñedo de uva para vino y $50,6 \%$ de uva de mesa.

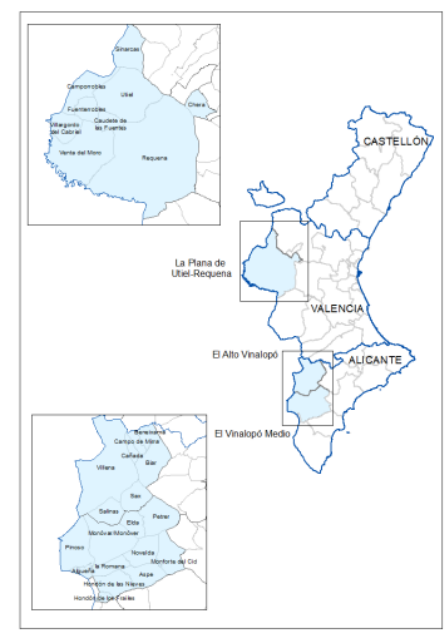

Figura 1: Área de estudio

\section{Metodología}

El análisis de las decisiones de los viticultores se ha realizado mediante un modelo de regresión logística multinivel (MLR) multinomial, utilizando el paquete estadístico IBM SPSS statistics 19.0 a través del procedimiento GLMM o modelos mixtos categóricos, con un método de máxima verosimilitud. La utilización de este modelo entiende que la relación de las parcelas vitícolas con las variables socioeconómicas y de cambio climático se asemejan más entre las que están en el mismo municipio o comarca que en otros. En este sentido se plantea un modelo de tres niveles: parcela, municipio y comarca (Figura 2). La variable dependiente es categórica e identifica las decisiones tomadas por los viticultores representadas en los derechos de plantación de la parcela: Las categorías de esta variable son: 0 Abandonadas; 1 Nuevas plantaciones; 2 Replantación; 3 Arranque; 4 Mantenimiento.

Las variables dependientes utilizadas se distribuyen en los tres niveles: parcelas, que identifican las características estructurales de las explotaciones; municipio, que describen las condiciones socioeconómicas y los pagos recibidos de las ayudas de la PAC y comarcales, que identifican el grado de esfuerzo del sector a los fenómenos del cambio climático. 


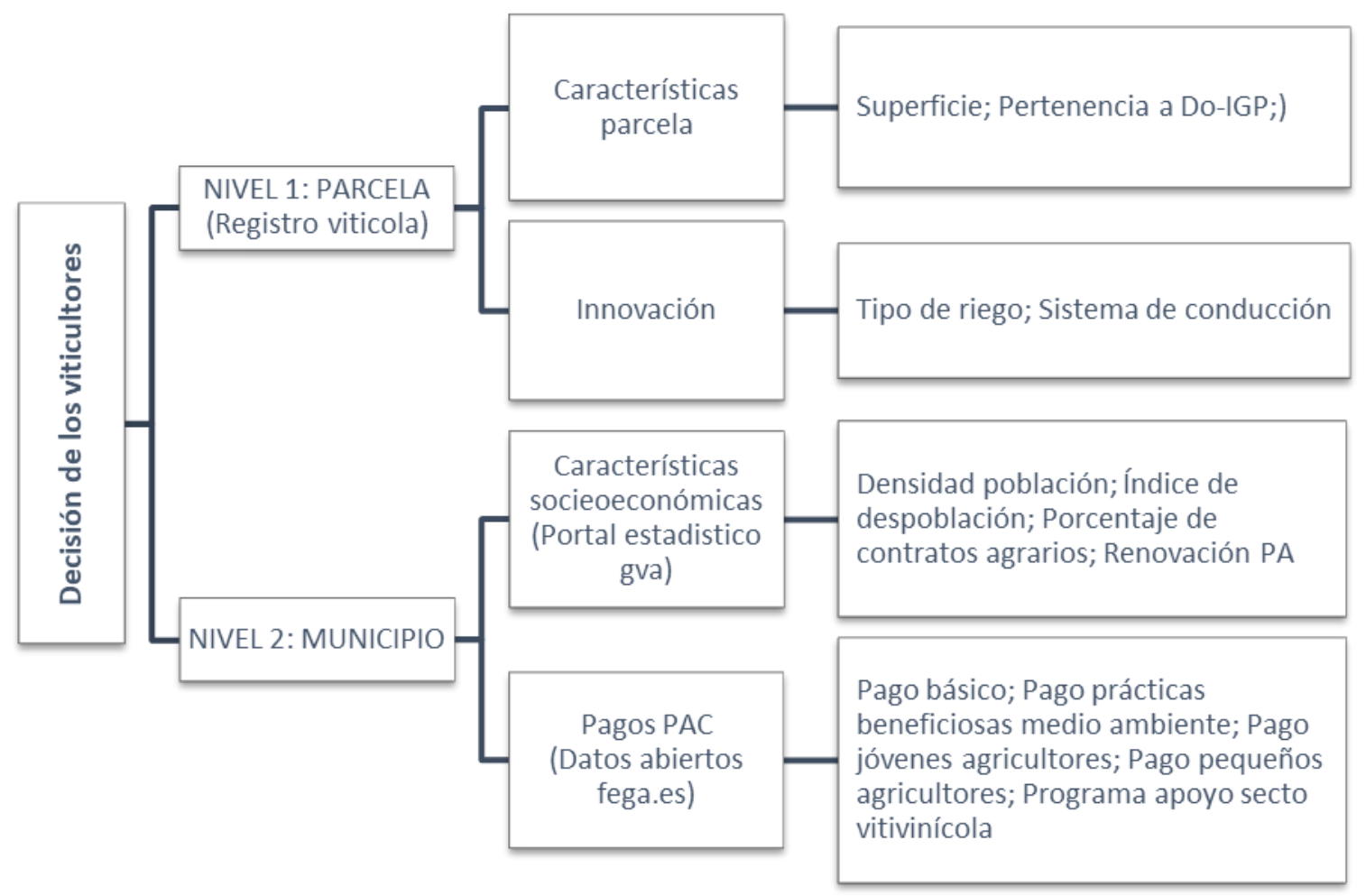

Figura 2: Variables del modelo multinivel según los niveles jerárquicos

\section{Resultados}

Los resultados del modelo nulo indican que hay variabilidad significativa en la medición de la decisión de los viticultores, lo que sugiere que es de interés examinar un modelo condicional que puede explicar parte de la variabilidad. La variabilidad es estadísticamente significativa tanto entre municipios, Wald $\mathrm{Z}=0.18$, $\mathrm{p}<.001$, como dentro de los municipios, Wald $\mathrm{Z}=1.13, \mathrm{p}<.001$. En la tabla 1 se presentan las estimaciones de los componentes de la varianza asociados con los efectos fijos y aleatorios de los tres parámetros.

Según estas estimaciones, la variabilidad entre los municipios representa el $(0,175) /(0,175+1,132)=$ 0,1343, o 13,43\% de la variabilidad total. Es decir, de la variabilidad total de la variable dependiente, aproximadamente un $13 \%$ corresponde a la diferencia entre las medias de los municipios.

Tabla1. Modelo Nulo para la estimación de la respuesta de los viticultores

\begin{tabular}{|l|c|c|c|c|}
\hline Efecto & \multirow{2}{*}{ Estimación } & \multirow{2}{*}{ Error típico } & \multicolumn{2}{|c|}{ Intervalo de confianza 95\% } \\
\cline { 4 - 5 } & & Límite inferior & Límite superior \\
\hline $\begin{array}{l}\text { Efectos fijos } \\
\text { Intercepto }\end{array}$ & $2.99 * * *$ & 0.083 & 2.81 & 3.16 \\
\hline Efectos aleatorios & & & & \\
Varianza entre-municipios & $0.18 * * *$ & 0.051 & 0.098 & 0.312 \\
Varianza dentro-municipio & $1.13 * * *$ & 0.006 & 1.120 & 1.145 \\
\hline
\end{tabular}

$* * * \mathrm{P}<.001$

En el modelo siguiente (Modelo 1) se han incluido los variables incluidas en la Figura 2. El número de parámetros se incrementa de 3 a 10. En la tabla 2 se muestran los resultados y se observa que la varianza entre municipios es significativa.

En la Tabla 3 se incluye los parámetros de los efectos fijos, mostrando que las variables de la estructura de las parcelas, tanta referente al tamaño (H11) como técnicas de innovación (H14, H120; H13) son significativas, y tienen un efecto negativo sobre la variable dependiente, excepto el sistema de conducción de las viñas. Las variables socioeconómicas del municipio significativas son el índice de despoblación (H31) y el porcentaje medio de contratos en el sector agrario (H32). De los pagos de las ayudas de la PAC, los más significativos son el pago a prácticas beneficiosas con el medio ambiente (PagoMA/ha), y con un grado de significación del 0.05 el Pago Básico, no siendo significativos el Pago a jóvenes agricultores y a pequeños agricultores. 
Tabla 2: Resultado de la varianza para el modelo 1.

\begin{tabular}{|l|r|r|r|r|r|r|}
\hline \multicolumn{9}{|c|}{ Parámetro } & Estimación & Error típico & Wald Z & \multicolumn{1}{c|}{ Sig. } & $\begin{array}{c}\text { Límite } \\
\text { inferior }\end{array}$ & $\begin{array}{c}\text { Límite } \\
\text { superior }\end{array}$ \\
\hline Residuos & 0.7851 & 0.004 & 180.981 & 0.000 & 0.776649 & 0.793654 \\
\hline Intersección & 0.2435 & 0.069 & 3.497 & 0.000 & 0.139005 & 0.0426495 \\
\hline
\end{tabular}

Tabla 3: Parámetros de los efectos fijos en el modelo 1

\begin{tabular}{|c|c|c|c|c|c|c|c|}
\hline Parámetro & Estimación & $\begin{array}{l}\text { Error } \\
\text { típico }\end{array}$ & gl & $\mathrm{t}$ & Sig. & $\begin{array}{l}\text { Límite } \\
\text { inferior }\end{array}$ & $\begin{array}{l}\text { Límite } \\
\text { superior }\end{array}$ \\
\hline Tipo de riego & -0.310244 & 0.007936 & 65502.259 & -39.095 & 0.000 & -0.325798 & -0.294689 \\
\hline Tamaño de parcela & -0.130028 & 0.009956 & $\begin{array}{r}65528 . \\
395\end{array}$ & -13.060 & 0.000 & -0.149542 & -0.110514 \\
\hline $\begin{array}{l}\text { Pertenencia } \\
\text { DO_IGP }\end{array}$ & -1.539529 & 0.011790 & 65534.990 & -130.575 & 0.000 & -1.562638 & -1.516419 \\
\hline $\begin{array}{l}\text { Afiliación al sector } \\
\text { agrario }\end{array}$ & 1.986746 & 0.218544 & 25.295 & 7.994 & 0.000 & 1.475162 & 2.498330 \\
\hline Pago viña/ha viñedo & 0.000009 & 0.000003 & 9925.966 & 2.734 & 0.011 & 0.000002 & 0.000001 \\
\hline $\begin{array}{ll}\text { Índice } & \text { de } \\
\text { despoblación } & \end{array}$ & 0.956891 & 0.318575 & 25.920 & 3.004 & 0.006 & 0.301953 & 1.611828 \\
\hline $\begin{array}{l}\text { Renovación Pobl. } \\
\text { activa }\end{array}$ & 0.764442 & 0.319284 & 24.976 & 2.394 & 0.024 & 0.10683 & 1.422052 \\
\hline Pago Básico/ha & -0.000103 & 0.000048 & 26.210 & -2.100 & 0.046 & -0.000203 & -0.000002 \\
\hline Pago Jóvenes/ha & -0.000821 & 0.000731 & 25.020 & -1.122 & 0.272 & -0.002327 & 0.000685 \\
\hline Pago Pequeños/ha & 0.000257 & 0.000124 & 26.214 & 2.074 & 0.048 & 0.000002 & 0.000513 \\
\hline $\begin{array}{l}\text { Pago Medidas } \\
\text { Medioambiente/ha }\end{array}$ & 0.000227 & 0.000049 & 26.416 & 4.581 & 0.000 & 0.000125 & 0.000329 \\
\hline $\begin{array}{l}\text { Sistema } \\
\text { conducción }\end{array}$ & 0.595408 & 0.006948 & 65309.09 & 85.3698 & 0.000 & 0.581791 & 0.609026 \\
\hline
\end{tabular}

\section{Conclusiones}

El modelo multinivel aplicado para predecir la decisión de los viticultores (mantener de la explotación, nuevas plantaciones, replantar, arrancar o abandonar) indica que tienen un efecto negativo la superficie de las explotaciones, el tipo de riego y la pertenencia a las DO, en cambio tienen un efecto positivo el sistema de conducción. Las características socioeconómicas de los municipios tienen efecto positivo en la decisión de los viticultores, principalmente el porcentaje de contratos en el sector agrario, pero también el riesgo de despoblación y la renovación de la población activa. Las ayudas recibidas de la PAC que tienen condicionado las decisiones de los viticultores son principalmente los Pagos a prácticas respetuosas con el medio ambiente y el Pago único, no siendo significativas los Pagos a jóvenes y pequeños agricultores. Por tanto, la innovación en el sistema de conducción de las viñas y los Pagos a las prácticas sostenibles, junto con las condiciones socioeconómicas de los municipios condicionan positivamente a los viticultores, y las características de las explotaciones lo hacen de forma negativa. En futuras investigaciones se deberían incorporar variables como los precios de mercado.

\section{Agradecimientos}

Esta investigación ha sido apoyada por la beca RTI2018-093791-B-C21 y RTI2018-093791-B-C22 financiada por el Ministerio de Ciencia (España) y el Fondo Europeo de Desarrollo Regional

\section{Bibliografía}

Cortignani, R. and S. Severini. (2011). "An extended PMP model to analyze farmers' adoption of deficit irrigation under environmental payments". Spanish Journal of Agricultural Research 9: 1035-1046.

Engler, A. and R. Toledo. (2010). "An analysis of factors affecting the adoption of economic and productive data recording methods of Chilean farmers". Ciencia e Investigación Agraria 37: 101-109. 
Giannoccaro, G. and J. Berbel. (2011). "Influence of the common agricultural policy on the farmer's intended decision on water use". Spanish Journal of Agricultural Research 9: 1021-1034.

Montagut, X. and F. Dogliotti. (2008). Alimentos globalizados: soberanía alimentaria y comercio justo. $2^{\mathrm{a}}$ edición, Icaria Editorial, Barcelona, Spain.

Naiggolan, D., M. Termansen, M.S. Reed, E.D. Cebollero and K. Hubacek. (2013). "Farmer typology, future scenarios and the implications for ecosystem service provision: a case study from southeastern Spain”. Regional Environmental Change 13: 601-614. 